\section{Tofacitinib in steroid-dependent relapsing polychondritis}

Relapsing polychondritis (RP) is a rare progressive inflammatory condition involving cartilaginous structures, predominantly those of the ears, nose and laryngotracheobronchial tree. The McAdam's diagnostic criteria require meeting three out of six of the following: recurrent chondritis of both auricles, nonerosive inflammatory polyarthritis, chondritis of nasal cartilages, ocular inflammation (conjunctivitis, keratitis, scleritis and/or uveitis), chondritis of respiratory tract, cochlear and/or vestibular damage (neurosensory hearing loss, tinnitus or vertigo). ${ }^{1}$ Systemic corticosteroids remain the mainstay of treatment for RP. Immunosuppressive agents (eg, methotrexate, azathioprine, dapsone, cyclophosphamide, etc) have been frequently used for the treatment of more severe and steroid-resistant manifestations of RP. The administration of biologics in patients with RP has been also described. ${ }^{23}$

In the recently published French multicentre retrospective cohort study, Moulis et al reported 41 patients with RP treated with 105 biological agents, including tumour necrosis factor (TNF) inhibitors, tocilizumab, anakinra, rituximab and abatacept. ${ }^{4}$ The reasons for initiating biological therapy were corticosteroid dependence, corticosteroid resistance or intolerance to methotrexate. Within the first six months of treatment, overall response (ie, at least partial clinical improvement) rate was $62.9 \%$. However, only $19.0 \%$ of patients achieved a complete response defined by no clinical activity. Response rates for TNF inhibitors, tocilizumab and rituximab (63.3\%-71.4\%) were numerically higher compared with those for abatacept and anakinra $(50.0 \%-53.3 \%)$. The results of the retrospective study suggested differences in efficacies depending on the organ involvement, that is, tocilizumab and TNF inhibitors, particularly adalimumab, were the most effective biologics for nasal/auricular chondritides and joint inflammation, respectively. Notably, steroid-sparing effect was modest (median daily corticosteroid dose was reduced by $5 \mathrm{mg}$ of prednisone equivalent), and nearly three-quarters of patients finally discontinued biologics due to insufficient efficacy (34.1\%), loss of efficacy (18.1\%) or adverse drug reactions $(20.9 \%)$.

Tofacitinib belongs to a new class of the Janus kinase inhibitors. It is approved for the treatment of rheumatoid arthritis, psoriasis and psoriatic arthritis. Current evidence suggests that tofacitinib may be also effective in patients with the other inflammatory diseases, such as ulcerative colitis, atopic dermatitis and alopecia. We observed a 39-year-old female patient with a 6-year history of $\mathrm{RP}$ that manifested by progressive nasal chondritis with saddle nose

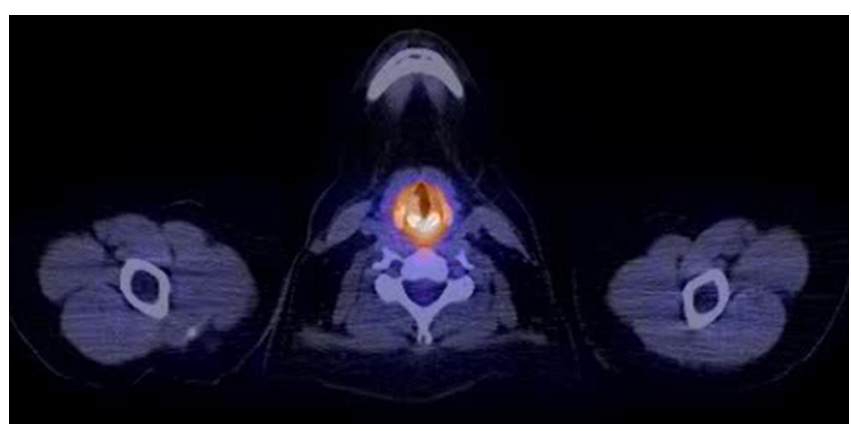

Figure 1 Uptake of tracer in laryngeal and tracheal cartilages on positron emission tomography/CT in patient with relapsing polychondritis. deformity, recurrent arthritis and scleritis, involvement of laryngeal and tracheal cartilages (figure 1), low-grade fever and laboratory signs of inflammation (high erythrocyte sedimentation rate, C-reactive protein and rheumatoid factor). Diagnosis of RP was established according to the presence of four out of six McAdam's criteria. Treatment with medium-dose $(30 \mathrm{mg} /$ day $)$ prednisone was initially effective. For steroid tapering, methotrexate, azathioprine, mycophenolate mofetil and dapsone were administered sequentially. However, all immunomodulators were discontinued within a relatively short period of time as a result of adverse drug reactions (azathioprine and dapsone were stopped within 1 month due to anaemia and leucopenia) or persistent clinical and laboratory activity (methotrexate and mycophenolate mofetil were discontinued within 3-4 months due to clinical deterioration after prednisone tapering to $15 \mathrm{mg} /$ day). Despite medium-dose to high-dose (up to $40 \mathrm{mg} /$ day) corticosteroids, the disease showed a progressive course with recurrent episodes of acute respiratory distress and a persistent laboratory activity. The patient rejected treatment with any parenteral biologics. However, she gave an informed consent for an off-label oral tofacitinib administration. This agent was chosen given its action on the intracellular pathways responsible for triggering the inflammatory response. Treatment with tofacitinib $10 \mathrm{mg}$ daily rapidly induced a clinical decrease in disease activity with corticosteroid tapering. At 12 months of treatment, she was in stable clinical remission and free from corticosteroids, while CT scanning showed an improvement of laryngeal wall thickening.

$\mathrm{RP}$ is an immune-mediated disease with poorly understood pathogenesis. Human and animal studies suggest that a complex cytokine network orchestrates the recruitment of infiltrating cells in RP lesions. ${ }^{5}$ Unlike biological treatments that target one cytokine pathway in the inflammatory network, tofacitinib inhibits the intracellular effects of several inflammatory mediators, resulting in downregulation of the immune and inflammatory response. ${ }^{6}$

Moulis et al showed at least partial efficacy of biological therapy in a significant proportion of patients with refractory RP, despite low complete response rate and modest steroidsparing effect. There was no single drug having apparent advantages in efficacy and safety over other biologics. However, infliximab and adalimumab should be preferred among the TNF antagonists. Tocilizumab was highly effective for almost all features of RP, but with a relative high rate of withdrawal due to adverse drug reactions. Our case report suggests that tofacitinib may constitute an additional therapeutic option for selected patients with RP. However, its efficacy and safety in RP versus non-biological or biological disease-modifying antirheumatic drugs should be confirmed in well-controlled prospective head-to-head studies.

\author{
Alexey D Meshkov, ${ }^{1}$ Pavel I Novikov, ${ }^{1}$ Evgeny V Zhilyaev, ${ }^{2}$ \\ Ilia David J llevsky, ${ }^{3}$ Sergey V Moiseev ${ }^{1}$ \\ ${ }^{1}$ Tareev Clinic of Internal Diseases, Sechenov First Moscow State Medical University, \\ Moscow, Russia \\ ${ }^{2}$ European Medical Center, Moscow, Russia \\ ${ }^{3}$ Municipal Polycinic \#64, Moscow, Russia
}

Correspondence to Professor Sergey V Moiseev, Tareev Clinic of Internal Diseases, Sechenov First Moscow State Medical University Moscow Russia ; clinpharm@mtu-net.ru

Handling editor Josef S Smolen

Contributors All authors participated in the preparation of the manuscript. Competing interests None declared.

Patient consent Not required. 
Provenance and peer review Not commissioned; internally peer reviewed.

(c) Article author(s) (or their employer(s) unless otherwise stated in the text of the article) 2019. All rights reserved. No commercial use is permitted unless otherwise expressly granted.

\section{A) Check for updates}

To cite Meshkov AD, Novikov PI, Zhilyaev EV, et al. Ann Rheum Dis 2019;78:e72.

Received 10 April 2018

Revised 23 April 2018

Accepted 24 April 2018

Published Online First 3 May 2018

Ann Rheum Dis 2019;78:e72. doi:10.1136/annrheumdis-2018-213554

\section{REFERENCES}

1 McAdam LP, O'Hanlan MA, Bluestone R, et al. Relapsing polychondritis: prospective study of 23 patients and a review of the literature. Medicine 1976;55:193-215.

2 Leroux G, Costedoat-Chalumeau N, Brihaye B, et al. Treatment of relapsing polychondritis with rituximab: a retrospective study of nine patients. Arthritis Rheum 2009:61:577-82.

3 Moulis G, Sailler L, Pugnet G, et al. Biologics in relapsing polychondritis: a case series. Clin Exp Rheumatol 2013:31:937-9.

4 Moulis G, Pugnet G, Costedoat-Chalumeau N, et al. Efficacy and safety of biologics in relapsing polychondritis: a French national multicentre study. Ann Rheum Dis 2018;77:1172-8.

5 Arnaud L, Mathian A, Haroche J, et al. Pathogenesis of relapsing polychondritis: a 2013 update. Autoimmun Rev 2014;13:90-5.

6 Hodge JA, Kawabata TT, Krishnaswami S, et al. The mechanism of action of tofacitinib - an oral Janus kinase inhibitor for the treatment of rheumatoid arthritis. Clin Exp Rheumatol 2016;34:318-28. 